Here, also, a distinction was drawn between uncomplicated refractive errors, and symptomatic, mechanical, and motor anomalies of vision.

\title{
SUPPURATION AFTER CATARACT EXTRACTION SUCCESSFULLY TREATED
}

\author{
BY \\ E. W. Wood White and T. Harrison Butler, \\ BIRMINGHAM. \\ COVENTRY.
}

IT is only rarely that an eye which has definitely commenced to suppurate after a cataract operation can be saved, and we therefore feel that the following example should be placed on record.

E. H., aged 32 years, was admitted to the Birmingham Eye Hospital, and on April 17, 1917, the right lens was extracted by the combined operation. The patient squeezed badly and vitreous was lost. At the first dressing the eye was somewhat injected, and in spite of treatment this increased. On April 28 there was a line of pus along the lips of the incision, and a mass of septic exudate was seen leading from the wound well into the anterior chamber. An anaesthetic was administered and the wound thoroughly cauterized with the actual cautery. The hot wire was drawn along the line of the section and allowed to burn well into the anterior chamber till the aqueous was evacuated; it was used in the most drastic fashion. A sub-conjunctival injection of oxy-cyanide of mercury of the strength of $1: 5,000$ was given, and 10 cubic centimetres of anti-. streptococcal serum was injected under the skin of the abdomen. A culture was made from the lips of the incision and a pure growth of pneumococci was obtained.

The effect of this treatment was almost magical, and in a few days the eye was out of danger.

On September 29, the eye was examined and found to be free from any injection. The upper segment of the cornea showed a dense nebula like an exaggerated arcus senilis. There were some fine posterior synechiae and a dense capsule.

Eventually the capsule was needled and an acuity of 6/18 was obtained. In January, 1918, the left lens was extracted. Again the patient squeezed badly and vitreous presented. The lens was removed with the spoon, and more vitreous escaped. In spite of these drawbacks the ultimate vision was 6/18.

This case shows that it is always worth while to treat such a case however hopeless it may appear to be ; but we think that to be of any value the actual cautery must be employed with energy. 